\title{
Dynamic analysis of nonlocal-gradient elastic nano-beams resting on an elastic foundation
}

\author{
Jianshe Peng ${ }^{1, \text { a }}$, Liu Yang ${ }^{1,}$, , Fan Lin ${ }^{2}$ \\ ${ }^{1}$ School of Mechanical Engineering, Cheng Du University, Cheng Du, 610106, China \\ ${ }^{2}$ School of Mechanical Engineering and Automation, Xi Hua University, Cheng Du, 610039, China \\ apengjianshe2005@163.com, ${ }^{b} 184312526 @ Q Q . c o m$
}

Keywords: nano-beams, dynamic analysis, nonlocal elasticity theory, strain gradient theory, elastic foundation.

Abstract. Dynamic analysis of nonlocal-gradient elastic nano-beams resting on an elastic foundation is investigated in this paper. The nonlocal-gradient elastic beam model, which has two independent gradient coefficients, based on the classical nonlocal elasticity theory and strain gradient theory, can be interpreted the size effect. By employing Galerkin method, the nonlinear partial differential governing equation is decoupled into a set of nonlinear ordinary differential equations which are then solved using Runge-Kutta method. Influence of elastic foundation coefficients and two independent gradient coefficients on the dynamic response of the nano-beam are investigated.

\section{Introduction}

Nano-beams are important building blocks in nano-electro-mechanical-systems (NEMS). The size scales associated with nanotechnology are too small to call the applicability of classical scale independent continuum models into question for NEMS structures because they cannot capture the small scale effect observed in both experiments and molecular dynamics simulations [1]. Size-dependent continuum theories have thus attracted increasing attention in modelling nano-structures and devices. Among these, nonlocal theory developed by Eringen [2, 3] has gained wide acceptance, which introduces the small scale effect through a spatial integral constitutive relation and allows for the stress at a point to be dependent on strains at all points in the elastic body. Gradient theory, another size-dependent continuum theory, was introduced by Aifantis and his coworkers [4] in the beginning of 1980s. A mixed nonlocal-gradient elasticity beam model with two independent gradient coefficients based on the classical nonlocal elasticity theory and strain gradient theory is proposed by Jun Shen and Xian Fang Li [5] to study wave propagation in single- and double- walled carbon nanotubes. Abu-Salih and Elata [6] analyzed the electromechanical buckling of a pre-stressed layer bonded to an elastic foundation. The effect of stiffening and softening elastic foundations on the post buckling behavior of the system is discussed.

In this paper, the governing equation of electrostatically actuated nano-beams resting on an elastic foundation has been obtained based on the nonlocal-gradient elastic theory, and then solved by Galerkin theory and Runge Kutta method. Numerical results show that the deflection response and frequency response of nano-beams are all affected by elastic foundation coefficients and two independent gradient coefficients.

\section{Theoretical formulation}

Linear foundation term is added to the equation of motion given in Ref. [5,7]. Hence, the governing equation of a nano-beam is

$$
E I\left(1-l_{2}^{2} \frac{\partial^{2}}{\partial \bar{x}^{2}}\right) \frac{\partial^{4} \bar{w}}{\partial \bar{x}^{4}}+\rho A\left(1-l_{1}^{2} \frac{\partial^{2}}{\partial \bar{x}^{2}}\right) \frac{\partial^{2} \bar{w}}{\partial \bar{t}^{2}}+k \bar{w}=\left(1-l_{1}^{2} \frac{\partial^{2}}{\partial \bar{x}^{2}}\right) q
$$

where $\bar{w}$ is the resulting transverse displacement of the nano-beam, $\bar{x}$ is the coordinate value along the axial direction, $\bar{t}$ is the time, $l_{1}$ and $l_{2}$ are the independent gradient coefficients, respectively, $E$ 
is the Young's modulus, $I$ is the second moment of inertia of the beam, $\rho$ is the mass density, $A$ is the cross-sectional area, $L$ is the length, and $q$ is the load, respectively. $k$ is the linear coefficient of the foundation.

Ignoring the fringing field effect, the electrostatic force per unit length of the beam is [8]

$$
q(\bar{t})=\frac{\varepsilon_{0} V^{2} b}{2(g-\bar{w})^{2}}
$$

where $\varepsilon_{0}$ is the vacuum dielectric constant, $V$ is the voltage, $b$ is the width, $g$ is the initial gap between the substrate and the beam.

It can be derived from equations (1) and (2) that the governing equation of the nano-beam is

$$
E I\left(1-l_{2}^{2} \frac{\partial^{2}}{\partial \bar{x}^{2}}\right) \frac{\partial^{4} \bar{w}}{\partial \bar{x}^{4}}+\rho A\left(1-l_{1}^{2} \frac{\partial^{2}}{\partial \bar{x}^{2}}\right) \frac{\partial^{2} \bar{w}}{\partial \bar{t}^{2}}+k \bar{w}=\left(1-l_{1}^{2} \frac{\partial^{2}}{\partial \bar{x}^{2}}\right) \frac{\varepsilon_{0} V^{2} b}{2(g-\bar{w})^{2}}
$$

The dimensionless quantities are introduced to facilitate the following theoretical formulations

$$
x=\frac{\bar{x}}{L}, t=\omega \bar{t}, \Omega=\frac{\omega}{\omega_{L}}, \omega_{L}=\frac{1}{L^{2}} \sqrt{\frac{E I}{\rho A}}, w=\frac{\bar{w}}{g}, \alpha_{1}=\frac{l_{1}}{L}, \alpha_{2}=\frac{l_{2}}{L}, \alpha_{3}=\frac{k L^{4}}{E I}, \beta=\frac{\varepsilon_{0} V^{2} b L^{4}}{2 E I g^{3}} .
$$

Eq. (3) can then be rewritten in dimensionless form as

$$
\begin{aligned}
& \alpha_{2}^{2}(1-w)^{4} \frac{\partial^{6} w}{\partial x^{6}}-(1-w)^{4} \frac{\partial^{4} w}{\partial x^{4}}+\Omega^{2} \alpha_{1}^{2}(1-w)^{4} \frac{\partial^{4} w}{\partial t^{2} \partial x^{2}}-\Omega^{2}(1-w)^{4} \frac{\partial^{2} w}{\partial t^{2}}+\alpha_{3}(1-w)^{4} w \\
& =\beta\left[\alpha_{1}^{2}\left(\frac{\partial w}{\partial x}\right)^{2}-\alpha_{1}^{2}(1-w) \frac{\partial^{2} w}{\partial x^{2}}-(1-w)^{2}\right] .
\end{aligned}
$$

\section{Solution method}

The deflection of the nano-beam $w(x, t)$, can be separated into temporal and spatial by function $u(t)$ and $q(x)$, respectively, in the form of a series of products

$$
w(x, t)=u_{i}(t) q_{i}(x),
$$

where $u_{i}(t)$ is the ith generalized coordinate and $q_{i}(x)$ is the ith linear un-damped mode shape of the straight nano-beam.

The mode shape for the clamped-clamped beam takes the form of

$$
q_{i}(x)=1-\cos 2 i \pi x \text {. }
$$

Substituting Eq. (6) into Eq. (5) then applying Galerkin method gives

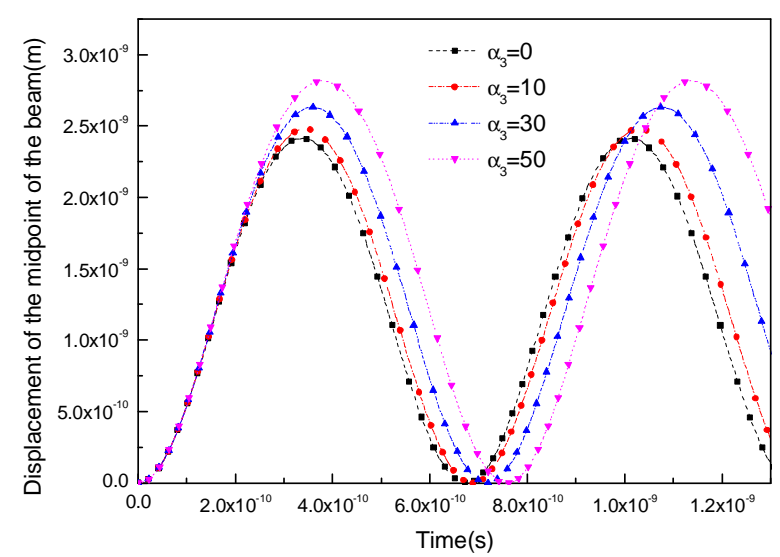

Fig.1 Mid-span deflection for various $\alpha_{3}$ values $\left(\alpha_{1}=0.2, \alpha_{2}=0.1\right.$, Voltage $\left.=5 \mathrm{~V}\right)$ 


$$
\begin{aligned}
& u_{i}^{(2)} \Omega^{2}\left[\begin{array}{l}
\alpha_{1}^{2} \int_{0}^{1} q_{i} q_{i}^{(2)} d x-4 u_{i} \alpha_{1}^{2} \int_{0}^{1} q_{i}^{2} q_{i}^{(2)} d x+6 u_{i}^{2} \alpha_{1}^{2} \int_{0}^{1} q_{i}^{3} q_{i}^{(2)} d x-4 u_{i}^{3} \alpha_{1}^{2} \int_{0}^{1} q_{i}^{4} q_{i}^{(2)} d x \\
+u_{i}^{4} \alpha_{1}^{2} \int_{0}^{1} q_{i}^{5} q_{i}^{(2)} d x-\int_{0}^{1} q_{i}^{2} d x+4 u_{i} \int_{0}^{1} q_{i}^{3} d x-6 u_{i}^{2} \int_{0}^{1} q_{i}^{4} d x+4 u_{i}^{3} \int_{0}^{1} q_{i}^{5} d x-u_{i}^{4} \int_{0}^{1} q_{i}^{6} d x
\end{array}\right] \\
& +u_{i}\left[\alpha_{2}^{2} \int_{0}^{1} q_{i} q_{i}^{(6)} d x-\int_{0}^{1} q_{i} q_{i}^{(4)} d x+\beta \alpha_{1}^{2} \int_{0}^{1} q_{i} q_{i}^{(2)} d x-2 \beta \int_{0}^{1} q_{i}^{2} d x+\alpha_{3} \int_{0}^{1} q_{i}^{2} d x\right] \\
& +u_{i}^{2}\left[-4 \alpha_{2}^{2} \int_{0}^{1} q_{i}^{2} q_{i}^{(6)} d x+4 \int_{0}^{1} q_{i}^{2} q_{i}^{(4)} d x-\beta \alpha_{1}^{2} \int_{0}^{1} q_{i}\left(q_{i}^{\prime}\right)^{2} d x-\beta \alpha_{1}^{2} \int_{0}^{1} q_{i}^{2} q_{i}^{(2)} d x+\beta \int_{0}^{1} q_{i}^{3} d x-4 \alpha_{3} \int_{0}^{1} q_{i}^{3} d x\right] \\
& +u_{i}^{3}\left[6 \alpha_{2}^{2} \int_{0}^{1} q_{i}^{3} q_{i}^{(6)} d x-6 \int_{0}^{1} q_{i}^{3} q_{i}^{(4)} d x+6 \alpha_{3} \int_{0}^{1} q_{i}^{4} d x\right] \\
& +u_{i}{ }^{4}\left[-4 \alpha_{2}^{2} \int_{0}^{1} q_{i}^{4} q_{i}^{(6)} d x+4 \int_{0}^{1} q_{i}^{4} q_{i}^{(4)} d x-4 \alpha_{3} \int_{0}^{1} q_{i}^{5} d x\right] \\
& +u_{i}^{5}\left[\alpha_{2}^{2} \int_{0}^{1} q_{i}^{5} q_{i}^{(6)} d x-\int_{0}^{1} q_{i}^{5} q_{i}^{(4)} d x+\alpha_{3} \int_{0}^{1} q_{i}^{6} d x\right] \\
& +\beta \int_{0}^{1} q_{i} d x=0
\end{aligned}
$$

It has been solved by Runge-Kutta method, and the result is discussed at next section.

\section{Numerical results}

To validate the present analysis, the dynamic response of a nano-beam clamped at both ends under a step voltage is considered, with the following geometric and material properties [9] $L=130 \mathrm{~nm}$, $b=4 n \mathrm{~m}, h=3.5 \mathrm{~nm}, g=10 \mathrm{~nm}, E=186.6 \mathrm{Gpa}, \varepsilon_{0}=8.854 \times 10^{-12}$ Farads $/ \mathrm{m}$.

Figs.1-3 display the time histories of the mid-span deflection of the clamped-clamped nano-beam under different coefficients and step voltages. As shown in Fig. 1 with increasing the coefficient $\alpha_{3}$, midpoint deflection of the beam increases and natural frequency decreases. As shown in Fig. 2 with

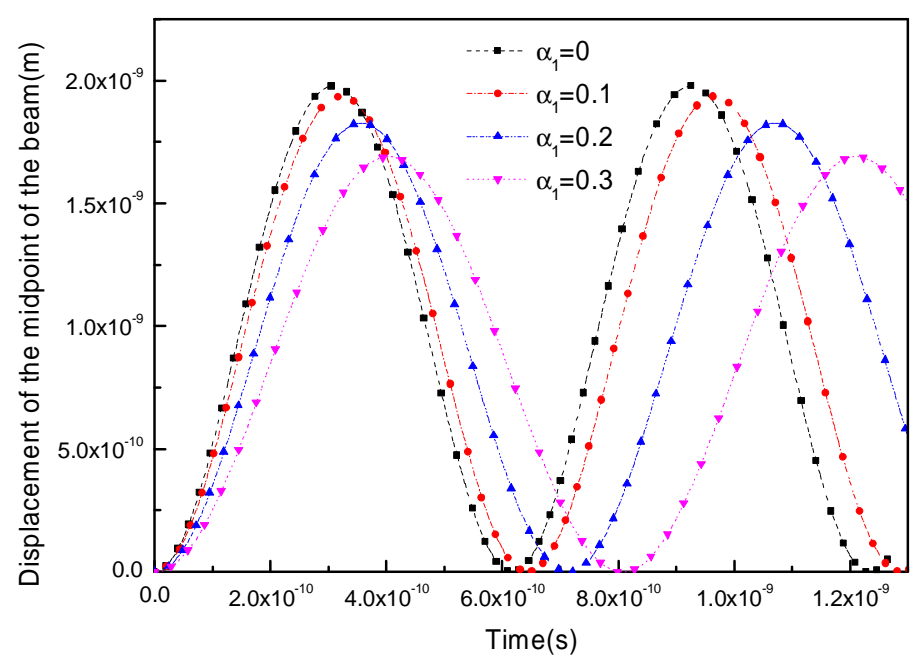

Fig. 2 Mid-span deflection for various $\alpha_{1}$ values $\left(\alpha_{2}=0.05, \alpha_{3}=10\right.$, Voltage $\left.=4 V\right)$ 


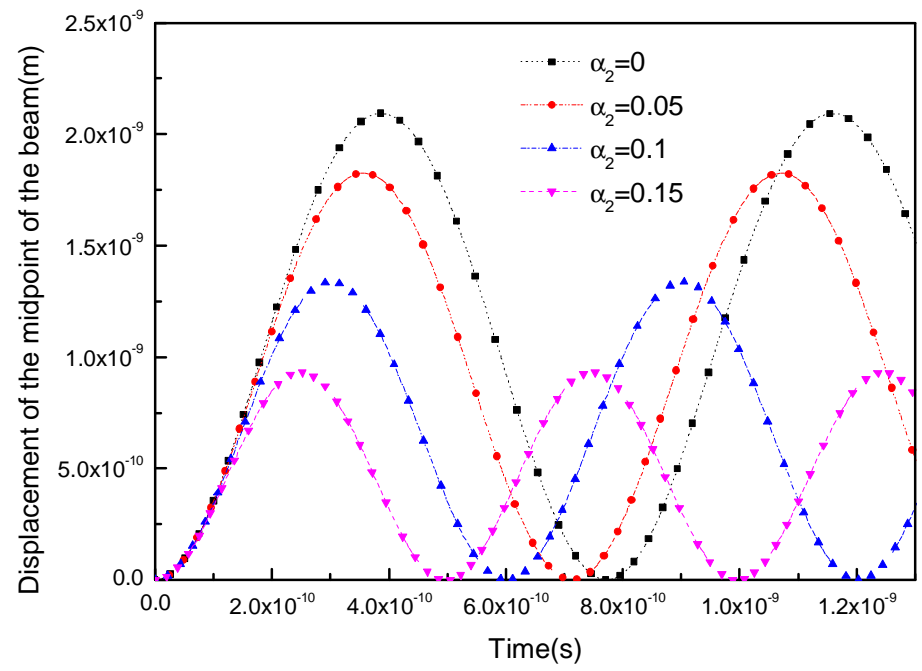

Fig.3 Mid-span deflection for various $\alpha_{2}$ values $\left(\alpha_{1}=0.2, \alpha_{3}=10\right.$, Voltage $\left.=4 \mathrm{~V}\right)$

increasing the gradient coefficient $\alpha_{1}$, midpoint deflection and natural frequency of the beam decreases. As shown in Fig.3 with increasing the gradient coefficient $\alpha_{2}$, midpoint deflection of the beam decreases and natural frequency increases.

\section{Conclusions}

The dynamic analysis of nano-beams resting on an elastic foundation is investigated in this paper based on the nonlocal-gradient elastic theory. The governing equation is solved by using Galerkin method and Runge-Kutta method. It is found from the numerical results that the midpoint deflection of the beam increases and natural frequency decreases with increasing the coefficient $\alpha_{3}$, the midpoint deflection and natural frequency of the beam decreases with increasing the gradient coefficient $\alpha_{1}$ and the midpoint deflection of the beam decreases and natural frequency increases with increasing the gradient coefficient $\alpha_{2}$. Results provide a reference for the choice of design and industrial applications of such nano-beams bonded to an elastic foundation.

\section{References}

[1] J. Yang, X .L. Jia and S. Kitipornchai: J. Phys. D: Appl Phys. Vol. 41 (2008), p. 035103.

[2] A. C. Eringen: J. Appl. Phys. Vol. 54 (1983), p. 4703-4710.

[3] A. C. Eringen: Nonlocal Continuum Field Theories (Springer, Berlin 2002).

[4] E. C. Aifantis: J. Mech Mater. Vol. 35 (2003), p. 259-280.

[5] J. Shen, X. F. Li. Scale Effect on Wave Propagation of Carbon Nanotubes [J]. Science and technology of west china. Vol.9 (2010).

[6] S. Abu-salih, D. Elata: NSTI Nanotech. Vol.2 (2004), p.223-226.

[7] G. Sari: Arab J Sci Eng. Vol. 38 (2013), p.1191-1199.

[8] W.C. Xie, H.P. Lee, S.P. Lim: J. Nonlinear Dyn. Vol. 31(2003), p.243-256.

[9] M. NikkhahBahrami, A. Ataei. A large deflection model for the dynamic pull-in analysis of electrostatically actuated nanobeams in presence of intermolecular surface forces [J]. Advanced research in physics and engineering. 